\title{
Exponential integrability of mappings of finite distortion
}

\author{
Tuomo Äkkinen and Kai Rajala
}

\begin{abstract}
We consider mappings with exponentially integrable distortion whose Jacobian determinants are integrable over the $n$-ball. We show that the boundary extensions of such mappings are exponentially integrable with bounds, and give examples to illustrate that there is not too much room for improvement. This extends the results of Beurling [2], and Chang and Marshall [3], [10] on analytic functions, and Poggi-Corradini and Rajala [14] on quasiregular mappings.
\end{abstract}

\section{Introduction}

A mapping $f: \Omega \rightarrow \mathbb{R}^{n}$, on a domain $\Omega \subset \mathbb{R}^{n}$ has finite distortion if the following conditions are fulfilled:

(a) $f \in W_{\text {loc }}^{1,1}\left(\Omega, \mathbb{R}^{n}\right)$,

(b) $J_{f}=\operatorname{det}(D f) \in L_{\mathrm{loc}}^{1}(\Omega)$,

(c) there exists a measurable $K_{f}: \Omega \rightarrow[1, \infty)$, so that for almost every $x \in \Omega$ we have

$$
|D f(x)|^{n} \leq K_{f}(x) J_{f}(x)
$$

where $|\cdot|$ is the operator norm. If $K_{f} \leq K<\infty$ almost everywhere, we say that $f$ is $K$-quasiregular. If $n=2$ and $K=1$, we recover complex analytic functions. See [15], [16] and [17] for the theory of quasiregular mappings, and [6], [7] for the theory of mappings of finite distortion.

We consider mappings $f: \mathbb{B}^{n} \rightarrow \mathbb{R}^{n}$ for which $f(0)=0$ and

$$
\int_{\mathbb{B}^{n}} J_{f} d x \leq \alpha_{n},
$$

where $\mathbb{B}^{n}$ is the unit $n$-ball with Lebesgue measure $\alpha_{n}$.

Mathematics Subject Classification (2010): 30C65.

Keywords: Mappings of finite distortion, exponential integrability, radial extension. 
Our main results are concerned with mappings with exponentially integrable distortion. More precisely, we assume that there are constants $\lambda, \mathcal{K}>0$ such that

$$
\int_{\mathbb{B}^{n}} \exp \left(\lambda K_{f}\right) d x \leq \mathcal{K} .
$$

We denote by $\mathcal{F}_{\lambda, \mathcal{K}}$ the class of mappings satisfying these assumptions.

Integrating the derivative over radial segments and applying polar coordinates, (1.1) and (1.2), it follows that if $f \in \mathcal{F}_{\lambda, \mathcal{K}}$, then $f$ has a radial limit $\bar{f}(\xi)$ at almost every boundary point $\xi \in \mathbb{S}^{n-1}$. See [1] for the existence of radial limits under milder assumptions. In this paper we prove an analog of known exponential integrability results for the boundary extension $\bar{f}$. We extend the results obtained for analytic functions in [2] and [3] and for quasiregular mappings in [14] to the class $\mathcal{F}_{\lambda, \mathcal{K}}$. We briefly recall these earlier results.

\subsection{Exponential integrability for analytic functions}

Let $\mathcal{F}_{1}$ be the class of analytic functions on the unit disc $\mathbb{D}$ with the above properties. Chang and Marshall proved the following sharp extension of an earlier result by Beurling [2].

Theorem A ([3], Corollary 1). We have

$$
\sup _{f \in \mathcal{F}_{1}} \int_{0}^{2 \pi} \exp \left(\left|\bar{f}\left(e^{i \theta}\right)\right|^{2}\right) d \theta<\infty .
$$

This result is sharp in the following sense: define, for $0<a<1$, the Beurling functions $B_{a}: \mathbb{D} \rightarrow \mathbb{C}$, so that

$$
B_{a}(z)=\log \left(\frac{1}{1-a z}\right) \log ^{-1 / 2}\left(\frac{1}{1-a^{2}}\right) .
$$

Then, for each $0<a<1, B_{a} \in \mathcal{F}_{1}$. Moreover, one can show that

$$
\lim _{a \rightarrow 1} \int_{0}^{2 \pi} \exp \left(\gamma\left|B_{a}\left(e^{i \theta}\right)\right|^{2}\right) d \theta=\infty
$$

for every $\gamma>1$. Essén [5] has generalized Theorem A, assuming instead of (1.1) the weaker condition $|f(\mathbb{D})| \leq \pi$.

\subsection{Exponential integrability for quasiregular mappings}

Let $n \geq 2$, and let $\mathcal{F}_{K}$ be the class of $K$-quasiregular mappings satisfying the above properties. Theorem A was generalized by Poggi-Corradini and Rajala [14] in the following form.

Theorem B ([14], Theorem 1.1). Let $n \geq 2$. Then

$$
\sup _{f \in \mathcal{F}_{K}} \int_{\mathbb{S}^{n-1}} \exp \left(\alpha|\bar{f}(\xi)|^{n /(n-1)}\right) d \xi<\infty,
$$


where

$$
\alpha=(n-1)\left(\frac{n}{2 K}\right)^{1 /(n-1)} .
$$

Theorem B is sharp in dimension 2 in the sense that

$$
\sup _{f \in \mathcal{F}_{K}} \int_{0}^{2 \pi} \exp \left(\gamma\left|\bar{f}\left(e^{i \theta}\right)\right|^{2}\right) d \theta=\infty
$$

for any $\gamma>1 / K$. This can be seen by composing the Beurling functions with a radial stretching, see Section 4. In higher dimensions, it is expected that the theorem is not sharp for any $K$. The proof of Theorem B in [14] follows the approach of Marshall [10] to Theorem A. A version of Theorem B for monotone functions was proved in [12].

\subsection{Exponential integrability for mappings of finite distortion}

The main result of this paper is the following counterpart of Theorems A and B for $\mathcal{F}_{\lambda, \mathcal{K}}$.

Theorem 1.1. Let $n \geq 2$ and $\lambda, \mathcal{K}>0$. There exists a constant $\alpha=\alpha(n, \lambda)>0$ such that

$$
\sup _{f \in \mathcal{F}_{\lambda, \mathcal{K}}} \int_{\mathbb{S}^{n-1}} \exp (\alpha|\bar{f}(\xi)|) d \xi<\infty .
$$

This is sharp in the sense that there exists $\hat{\alpha}=\hat{\alpha}(n, \lambda)>\alpha$ such that

$$
\sup _{f \in \mathcal{F}_{\lambda, \mathcal{K}}} \int_{\mathbb{S}^{n-1}} \exp (\hat{\alpha}|\bar{f}(\xi)|) d \xi=\infty .
$$

However, we do not know the best constant $\alpha$ in Theorem 1.1, even in dimension 2 . We discuss this issue in the next subsection.

\subsection{Estimate for level sets}

Marshall [10] gave a proof of Theorem A using an estimate of Beurling [2] on logarithmic capacity, together with sharp estimates established by Moser [11]. The proof of Theorem B in [14] uses similar ideas and in particular an "egg-yolk principle", discussed in Section 5 below, and an extension of Beurling's estimate to all dimensions. We state a simple consequence of this estimate. Denote

$$
F_{s}=\left\{\xi \in \mathbb{S}^{n-1}:|\bar{f}(\xi)| \geq s\right\}, \quad E_{t}=\left\{x \in \mathbb{B}^{n}:|f(x)|=t\right\}
$$

and

$$
\mathcal{A}_{n-1} f\left(E_{t}\right)=\int_{S^{n-1}(0, t)} \operatorname{card} f^{-1}(y) d \mathcal{H}^{n-1}(y)
$$


Theorem C ([14], Theorem 1.5). Suppose that $f$ is a $K$-quasiregular mapping in a neighborhood of $\overline{\mathbb{B}}^{n}$ such that $|f(x)| \leq M$ whenever $|x| \leq r<1$. Then, for every $s>M$,

$$
\mathcal{H}^{n-1}\left(F_{s}\right) \leq C \exp \left(-(n-1)\left(\frac{n \alpha_{n}}{2 K}\right)^{1 /(n-1)} \int_{M}^{s} \frac{d t}{\left(\mathcal{A}_{n-1} f\left(E_{t}\right)\right)^{1 /(n-1)}}\right),
$$

where $C=C(n, r, K)>0$.

This result allows one to estimate the level sets of the boundary extension $\bar{f}$ in the previous theorems, and it is sharp in dimension 2. We prove the following version with exponentially integrable distortion.

Theorem 1.2. Suppose that $f$ is a mapping of finite distortion in a neighborhood of $\overline{\mathbb{B}}^{n}$, satisfying (1.2), such that $|f(x)| \leq M$ whenever $|x| \leq r<1$. Then, for every $s>M$,

$$
\mathcal{H}^{n-1}\left(F_{s}\right) \leq C_{1} \exp \left(-C_{2}\left(\int_{M}^{s} \frac{d t}{\left(\mathcal{A}_{n-1} f\left(E_{t}\right)\right)^{1 /(n-1)}}\right)^{(n-1) / n}\right),
$$

where $C_{1}, C_{2}>0$ depend on $n$ and $\lambda$, and $C_{1}$ also depends on $r$ and $\mathcal{K}$.

Theorem 1.1 follows from this estimate. We do not know the best constant $C_{2}$ in Theorem 1.2, even in dimension 2. In Theorem $\mathrm{C}$, the best constant can be obtained by applying symmetrization methods to give sharp capacity estimates for the conformal $n$-capacity. In Theorem 1.2 one needs to work with weighted capacities, and it seems that symmetrization methods do not work here. Therefore, we have to use a different method that does not give the best constant.

\section{Proof of Theorem 1.1 assuming Theorem 1.2}

Weaker versions of Theorem A and Theorem B, with constants below the critical constant, are considerably easier to prove, using Theorem C. We demonstrate this well-known fact. Since analytic functions are 1-quasiregular mappings, we only consider Theorem B. First, recall that mappings in class $\mathcal{F}_{K}$ are equicontinuous. In particular, there exists a constant $r_{0}=r_{0}(n, K)>0$ such that

$$
|f(x)| \leq 1 \text { for every }|x| \leq r_{0},
$$

see [7]. We now claim Theorem B below the critical exponent. More precisely, we claim that

$$
\sup _{f \in \mathcal{F}_{K}} \int_{\mathbb{S}^{n-1}} \exp \left(\gamma|\bar{f}(\xi)|^{n /(n-1)}\right) d \xi<\infty
$$

whenever $\gamma<(n-1)(n /(2 K))^{1 /(n-1)}$. 
Recall the notation $F_{s}$ and $\mathcal{A}_{n-1} f\left(E_{t}\right)$ from Section 1.4. To prove (2.2), we apply Cavalieri's principle and write

$$
\int_{\mathbb{S}^{n-1}} \exp \left(\gamma|f(\xi)|^{n /(n-1)}\right) d \xi=n \alpha_{n}+\frac{\gamma n}{n-1} \int_{0}^{\infty} \mathcal{H}^{n-1}\left(F_{s}\right) e^{\gamma s^{1 /(n-1)}} s^{1 /(n-1)} d s .
$$

Therefore, it suffices to bound

$$
\int_{1}^{\infty} \mathcal{H}^{n-1}\left(F_{s}\right) e^{\gamma s^{1 /(n-1)}} s^{1 /(n-1)} d s .
$$

By Fatou's lemma we may assume that $f$ is defined on a neighborhood of $\overline{\mathbb{B}}^{n}$. Now if $s>1$ then by (2.1) and Theorem $\mathrm{C}$ we have

$$
\mathcal{H}^{n-1}\left(F_{s}\right) \leq C \exp \left(-(n-1)\left(\frac{n \alpha_{n}}{2 K}\right)^{1 /(n-1)} \int_{1}^{s} \frac{d t}{\left(\mathcal{A}_{n-1} f\left(E_{t}\right)\right)^{1 /(n-1)}}\right),
$$

for all $f \in \mathcal{F}_{K}$. Moreover, Hölder's inequality, change of variables and (1.1) yield

$$
(s-1)^{n /(n-1)} \leq \alpha_{n}^{1 /(n-1)} \int_{1}^{s} \frac{d t}{\left(\mathcal{A}_{n-1} f\left(E_{t}\right)\right)^{1 /(n-1)}},
$$

and thus

$$
\mathcal{H}^{n-1}\left(F_{s}\right) \leq C \exp \left(-\alpha(s-1)^{n /(n-1)}\right),
$$

where $\alpha=(n-1)(n /(2 K))^{1 /(n-1)}$. Therefore, since $\gamma<\alpha$,

$$
\begin{aligned}
& \sup _{f \in \mathcal{F}_{K}} \int_{1}^{\infty} \mathcal{H}^{n-1}\left(F_{s}\right) e^{\gamma s^{1 /(n-1)}} s^{1 /(n-1)} d s \\
& \leq C \int_{1}^{\infty} e^{\gamma s^{1 /(n-1)}-\alpha(s-1)^{n /(n-1)}} s^{1 /(n-1)} d s<\infty .
\end{aligned}
$$

In order to prove Theorems A and B one has to combine the arguments above with a method developed by Moser [11] to give a sharp version of Trudinger's inequality. See Section 5 for further discussion.

As discussed above, we are not able to prove Theorem 1.1 with the best possible constant. Therefore, the main difficulty in the proof is to establish Theorem 1.2. This is more difficult than in the case of quasiregular mappings. Once we have Theorem 1.2 at our disposal, Theorem 1.1 can be proved in a similar way as above.

Proof of Theorem 1.1. We repeat the steps above but with our new estimates. First, the equicontinuity property (2.1) holds also in the class $\mathcal{F}_{\lambda, \mathcal{K}}$ with $r_{0}=$ $r_{0}(n, \lambda, \mathcal{K})$, cf. [7]. Let $\alpha>0$ and $f \in \mathcal{F}_{\lambda, \mathcal{K}}$. By Cavalieri's principle we can write

$$
\int_{\mathbb{S} n-1} \exp (\alpha|\bar{f}(\xi)|) d \xi=n \alpha_{n}+\alpha \int_{0}^{\infty} \mathcal{H}^{n-1}\left(F_{s}\right) e^{\alpha s} d s
$$

and thus it suffices to bound

$$
\int_{1}^{\infty} \mathcal{H}^{n-1}\left(F_{s}\right) e^{\alpha s} d s .
$$


Now if $s>1$, then by equicontinuity and Theorem 1.2 we have

$$
\mathcal{H}^{n-1}\left(F_{s}\right) \leq C_{1} \exp \left(-C_{2}\left(\int_{1}^{s} \frac{d t}{\left(\mathcal{A}_{n-1} f\left(E_{t}\right)\right)^{1 /(n-1)}}\right)^{(n-1) / n}\right) .
$$

Moreover, (2.3) holds under our assumptions, and thus

$$
\mathcal{H}^{n-1}\left(F_{s}\right) \leq C_{1} \exp \left(-C_{2} \alpha_{n}^{-1 / n}(s-1)\right) .
$$

Combining the estimates, we have

$$
\sup _{f \in \mathcal{F}_{\lambda, \mathcal{K}}} \int_{1}^{\infty} \mathcal{H}^{n-1}\left(F_{s}\right) e^{\alpha s} d s \leq C \int_{1}^{\infty} e^{\alpha s-C_{2} \alpha_{n}^{-1 / n}(s-1)} d s<\infty
$$

if $\alpha<C_{2} \alpha_{n}^{-1 / n}$.

\section{Proof of Theorem 1.2}

\subsection{Symmetrization and weighted modulus}

We first discuss the methods used to prove Theorems $\mathrm{C}$ and 1.2. We will apply modulus (or capacity) estimates. Let $\Gamma$ be a family of paths in $\mathbb{R}^{n}$. Let $\rho: \mathbb{R}^{n} \rightarrow$ $[0, \infty]$ be a Borel measurable function. We say that $\rho$ is admissible for $\Gamma$, or $\rho \in \operatorname{Adm}(\Gamma)$, if

$$
\int_{\gamma} \rho d s \geq 1
$$

for all rectifiable $\gamma \in \Gamma$. If $\omega$ is a non-negative measurable function, then the weighted modulus $\operatorname{Mod}_{\omega}(\Gamma)$ is

$$
\operatorname{Mod}_{\omega}(\Gamma)=\inf _{\rho \in \operatorname{Adm}(\Gamma)} \int_{\mathbb{R}^{n}} \rho^{n}(x) \omega(x) d x .
$$

If $\omega \equiv 1$, then $\operatorname{Mod}(\Gamma)=\operatorname{Mod}_{\omega}(\Gamma)$ is the conformal modulus.

Let $0<r<1$ and $M=\max _{|x| \leq r}|f(x)|$. Consider the modulus of the family $\Gamma$ of paths connecting $B^{n}(0, r)$ to $F_{s}$. Define

$$
\rho(x)=\left(\int_{M}^{s} \frac{d t}{\left(\mathcal{A}_{n-1} f\left(E_{t}\right)\right)^{1 /(n-1)}}\right)^{-1} \frac{\|D f(x)\|}{\left(\mathcal{A}_{n-1} f\left(E_{t}\right)\right)^{1 /(n-1)}},
$$

when $|f(x)|=t \in(M, s)$ and $\rho(x)=0$ otherwise. Then $\rho$ is admissible for $\Gamma$ outside a negligible exceptional set. After a change of variables and an application of the distortion inequality for $f$, this implies

$$
\operatorname{Mod}_{1 / K}(\Gamma) \leq \int_{\mathbb{R}^{n}} \rho(x)^{n} d x=\left(\int_{M}^{s} \frac{d t}{\left(\mathcal{A}_{n-1} f\left(E_{t}\right)\right)^{1 /(n-1)}}\right)^{1-n}
$$


Therefore, Theorems $\mathrm{C}$ and 1.2 follow if a suitable lower bound for $\operatorname{Mod}_{1 / K}(\Gamma)$ is found. In the case of quasiregular mappings, this amounts to proving a lower bound for $\operatorname{Mod}(\Gamma)$. In this case, the lower bound

$$
\operatorname{Mod}(\Gamma) \geq \frac{n \alpha_{n}}{2}\left(\log \frac{C}{\mathcal{H}^{n-1}\left(F_{s}\right)^{1 /(n-1)}}\right)^{1-n}
$$

can be established using symmetrization methods. More precisely, (3.2) is proved by spherical cap symmetrization and it is sharp in the sense that the constant in front of the logarithm is the best possible. Theorem $\mathrm{C}$ follows by combining the estimates. See [14] and the references therein for details.

In the case of exponentially integrable $K_{f}$, one would also like to prove a sharp lower bound for $\operatorname{Mod}_{1 / K}(\Gamma)$ using symmetrization. It seems that symmetrization methods cannot be directly applied to the weighted modulus. Using the exponential integrability of $K_{f}$ one can, however, show that

$$
\operatorname{Mod}_{1 / K}(\Gamma) \geq C \varphi\left(\operatorname{Mod}^{*}(\Gamma)\right),
$$

where $\varphi(t)=t / \log (e+1 / t)$, and

$$
\operatorname{Mod}^{*}(\Gamma)=\inf _{\rho \in \operatorname{Adm}(\Gamma)} \int_{\mathbb{R}^{n}} \frac{\rho^{n}(x)}{\log (e+\rho(x))} d x .
$$

Now it follows from [4] that $\operatorname{Mod}^{*}(\Gamma)$ is reduced under spherical symmetrization. Therefore, we get

$$
\operatorname{Mod}^{*}\left(\Gamma_{0}\right) \geq C\left(\log \frac{1}{\mathcal{H}^{n-1}\left(F_{s}\right)^{1 /(n-1)}}\right)^{-n}
$$

Combining the estimates gives

$$
\mathcal{H}^{n-1}\left(F_{s}\right) \leq C_{1} \exp \left(-C_{2} I(s, M)^{(n-1) / n} \log ^{-1 / n}(e+I(s, M))\right),
$$

where

$$
I(M, s)=\int_{M}^{s} \frac{d u}{\left(\mathcal{A}_{n-1} f\left(E_{u}\right)\right)^{1 /(n-1)}} .
$$

This is weaker than what is claimed in Theorem 1.2. Therefore, we need to find an alternative method for proving Theorem 1.2. In what follows, we prove a better lower bound directly for the integral of $\rho^{n}$ using elementary chaining arguments and the exponential integrability of $K_{f}$.

\subsection{Proof of Theorem 1.2}

Let $f$ satisfy the assumptions of Theorem 1.2. Fix $0<r<1 / 2$ and set

$$
M=\max _{|x| \leq r}|f(x)|
$$


Let $s>M$, and let $\rho$ be the function in (3.1). Also, define $\hat{\rho}: \mathbb{R}^{n} \rightarrow[0, \infty]$,

$$
\hat{\rho}(x)=I(M, s)^{-1} \frac{J_{f}(x)^{1 / n}}{\left(\mathcal{A}_{n-1} f\left(E_{t}\right)\right)^{1 /(n-1)}},
$$

when $|f(x)|=t \in(M, s)$ and $\hat{\rho}(x)=0$ otherwise $(I(M, s)$ is defined in (3.3)). Then we have

$$
\rho(x) \leq \hat{\rho}(x) K_{f}(x)^{1 / n}
$$

almost everywhere by the distortion inequality. If $\gamma$ connects $B^{n}(0, r)$ to $F_{s}=$ $\left\{\xi \in \mathbb{S}^{n-1}:|\bar{f}(\xi)| \geq s\right\}$, we have

$$
\int_{\gamma} \rho d s \geq I(M, s)^{-1} \int_{f(\gamma)} \frac{1}{\left(\mathcal{A}_{n-1} f\left(E_{|x|}\right)\right)^{1 /(n-1)}} d s(x) \geq 1
$$

whenever the change of variables formula holds for the restriction of $f$ to $\gamma$. It is not difficult to see that the exceptional set does not affect any of the estimates below.

We also have

$$
\int_{\mathbb{R}^{n}} \hat{\rho}(x)^{n} d x=I(M, s)^{1-n} .
$$

Indeed, applying change of variables and polar coordinates, and recalling the definition of $\mathcal{A}_{n-1} f\left(E_{t}\right)$, we have

$$
\begin{aligned}
I(M, s)^{n} \int_{\mathbb{R}^{n}} \hat{\rho}(x)^{n} d x & =\int_{M \leq|y| \leq s} \frac{\operatorname{card} f^{-1}(y) d y}{\left(\mathcal{A}_{n-1} f\left(E_{|y|}\right)\right)^{n /(n-1)}} \\
& =\int_{M}^{s} \int_{S^{n-1}(0, t)} \frac{\operatorname{card} f^{-1}(y) d \mathcal{H}^{n-1}(y)}{\left(\mathcal{A}_{n-1} f\left(E_{t}\right)\right)^{n /(n-1)}} d t=I(M, s) .
\end{aligned}
$$

We claim that

$$
\int_{\mathbb{R}^{n}} \hat{\rho}(x)^{n} d x \geq C_{2}\left(\log \frac{C_{1}}{\mathcal{H}^{n-1}\left(F_{s}\right)}\right)^{-n},
$$

where $C_{1}$ and $C_{2}$ are as in the statement of the theorem. The theorem follows by combining (3.6) and (3.7). To prove (3.7), we will use a parametrization of $\mathbb{B}^{n}$ by "spherical coordinates" as follows. For $j \in \mathbb{N}$, set

$$
A_{j}=[0,1]^{n} \cap\left\{2^{-j} \leq x_{n} \leq 2^{-j+1}\right\}
$$

and divide $A_{j}$ into $2^{j(n-1)}$ closed cubes $Q_{i}^{j}$ of side length $2^{-j}$ with disjoint interiors. More precisely,

$$
Q_{i}^{j}=\left[i_{1} 2^{-j},\left(i_{1}+1\right) 2^{-j}\right] \times \ldots \times\left[i_{n-1} 2^{-j},\left(i_{n-1}+1\right) 2^{-j}\right] \times\left[2^{-j}, 2^{-j+1}\right],
$$

where $i=\left(i_{1}, \ldots, i_{n-1}\right) \in\left\{0, \ldots, 2^{j}-1\right\}^{n-1}$. Denote by $\mathcal{Q}^{j}$ the collection of $Q_{i}^{j}$ : $\mathrm{s}$ at level $j$, and set

$$
\mathcal{Q}=\cup_{j} \mathcal{Q}^{j}
$$


We also denote $Q_{0}^{0}=[0,1]^{n-1} \times[1,2]$, and

$$
G=[0,1]^{n-1} \times[0,2] .
$$

The top of a cube $Q_{i}^{j}$ is

$$
T\left(Q_{i}^{j}\right)=Q_{i}^{j} \cap\left\{x_{n}=2^{-j+1}\right\} .
$$

We say that $Q_{i}^{j}$ and $Q_{i^{\prime}}^{j+1}$ are consecutive if

$$
T\left(Q_{i^{\prime}}^{j+1}\right) \subset Q_{i}^{j} .
$$

Moreover, we say that a cube $Q_{i^{\prime}}^{l}$ is a descendant of $Q_{i}^{j}(l>j)$ if there is a sequence of consecutive cubes starting from $Q_{i}^{j}$ ending at $Q_{i^{\prime}}^{l}$, meaning any two cubes in order from the sequence are consecutive. With this terminology, each cube in $\mathcal{Q}$ has $2^{n-1}$ descendants at the next level.

The set $T\left(Q_{0}^{0}\right)$ can be mapped onto $T\left(Q_{i}^{j}\right)$ by scaling the first $n-1$ coordinates by a factor of $2^{-j}$ and composing with a translation. Denote such map by $\phi_{j}$ and let $l_{v}^{j}:[0,1] \rightarrow \mathbb{R}^{n}, l_{v}^{j}(t)=(1-t) \phi_{j}(v)+t \phi_{j+1}(v)$. Then

$$
\left\{l_{v}^{j}(t): v \in T\left(Q_{0}^{0}\right)\right\}
$$

is a family of line segments connecting $T\left(Q_{i}^{j}\right)$ to $T\left(Q_{i^{\prime}}^{j+1}\right)$ in $Q_{i}^{j}$. Adding these line segments together, we get piecewise linear paths connecting the tops of any two consecutive cubes. Recall that our goal is to estimate $\mathcal{H}^{n-1}\left(F_{s}\right)$ by the $n$-integral of $\hat{\rho}$. Applying the estimate below to the upper half space and lower half space, we may assume that $x_{n} \geq 0$ for every $x \in F_{s}$. There exists a universal constant $L>0$ and an $L$-bi-Lipschitz homeomorphism $h: G \rightarrow A^{+}(1 / 2,1)$, where

$$
A^{+}(1 / 2,1)=\left\{x \in \overline{\mathbb{B}^{n}(0,1)} \backslash \mathbb{B}^{n}(0,1 / 2): x_{n} \geq 0\right\},
$$

so that $h$ maps the bottom of $G$ onto the upper half of the unit sphere and the top onto the upper half of $S^{n-1}(0,1 / 2)$.

For each $k \in \mathbb{N}$, let $G_{k}=[0,1]^{n-1} \times\left[2^{-k}, 2+2^{-k}\right]$ and $h_{k}: G_{k} \rightarrow G, h_{k}(x)=$ $h\left(x_{1}, \ldots, x_{n-1}, x_{n}-2^{-k}\right)$. Moreover, define

$$
\mathcal{P}_{k}=\left\{Q \in \mathcal{Q}^{k}: h_{k}(Q) \cap F_{s} \neq \emptyset\right\}, \quad p_{k}=\operatorname{card} \mathcal{P}_{k} .
$$

Then, since the $h_{k}(Q)$ :s cover $F_{s}$, we have

$$
\mathcal{H}^{n-1}\left(F_{s}\right) \leq L^{n-1} p_{k} 2^{-k(n-1)},
$$

where $L$ is the bi-Lipschitz constant of $h$. Therefore, (3.7) follows if we show

$$
\int_{\mathbb{R}^{n}} \hat{\rho}(x)^{n} d x \geq C_{2}\left(\log \frac{C_{1}}{p_{k} 2^{-k(n-1)}}\right)^{-n} .
$$

Since $f$ has exponentially integrable distortion in a neighborhood of $\overline{\mathbb{B}}^{n}$ it is continuous in that neighborhood and thus uniformly continuous in $\overline{\mathbb{B}}^{n}$. Therefore, 
taking $k$ large enough, we may assume that $|f(x)| \geq s$ in $h_{k}(Q)$ for every $Q \in \mathcal{P}_{k}$. Fix $Q(\ell) \in \mathcal{P}_{k}, \ell=1, \ldots, p_{k}$, and let $\Gamma_{k}$ denote the family of paths in $G_{k}$ that connects the bottom of $Q(\ell)$ to the top of $G_{k}$, which are constructed from the line segments described above and line segments of length $2^{-k}$ parallel to $x_{n}$ axis connecting the top of $Q_{0}^{0}$ to the top of $G_{k}$. If $\gamma \in \Gamma_{k}$, then $h_{k} \circ \gamma$ connects $S^{n-1}(0,1 / 2)$ to $F_{s}$. If $h_{k}(\gamma)$ meets $S^{n-1}(0,1 / 2)$ at a point $z / 2$, we define $\gamma^{\prime}$ to be $h_{k} \circ \gamma$ outside $B^{n}(0,1 / 2)$, and the line segment $J$ connecting $z / 2$ and $r z$ in $B^{n}(0,1 / 2)$. Then $\gamma^{\prime}$ connects $F_{s}$ and $B^{n}(0, r)$. Therefore, by $(3.5)$,

$$
\int_{J} \rho d s+\int_{\gamma} \rho \circ h_{k} d s \geq L^{-1} \int_{\gamma^{\prime}} \rho d s \geq L^{-1},
$$

outside the exceptional set, where $L$ is the bi-Lipschitz constant of $h$.

Let $\left\{Q_{i_{j}}^{j}(\ell)\right\}_{j=0}^{k}$ be the sequence of consecutive cubes containing the paths $\gamma \in \Gamma_{k}$. With the parametrization introduced above and change of variables we have

$$
\int_{T\left(Q_{0}^{0}\right)} \int_{l_{v}^{j}} \rho \circ h_{k} d s d v \leq 2^{j(n-1)} \int_{Q_{i}^{j}} \rho \circ h_{k} d x .
$$

Thus integrating (3.10) over $T\left(Q_{0}^{0}\right)$, yields

$$
1 \leq C L \sum_{j=1}^{k} 2^{j(n-1)} \int_{Q_{i_{j}}^{j}(\ell)} \rho \circ h_{k}(x) d x+C L \int_{A(r, 1 / 2)} \frac{\rho(x)}{|x|^{n}} d x+\varepsilon(k),
$$

where $C$ depends only on $n, A(r, 1 / 2)=B^{n}(0,1 / 2) \backslash \bar{B}^{n}(0, r)$. The term $\varepsilon(k)>0$ comes from the line segments connecting $T\left(Q_{0}^{0}\right)$ to top of $G_{k}$. Moreover, $\lim _{k \rightarrow \infty} \varepsilon(k)$ $=0$ and thus it can be absorbed to the left hand side. Summing over $\ell$ gives

$$
p_{k} \leq C L \sum_{\ell=1}^{p_{k}} \sum_{j=1}^{k} 2^{j(n-1)} \int_{Q_{i_{j}}^{j}(\ell)} \rho \circ h_{k}(x) d x+p_{k} C L \int_{A(r, 1 / 2)} \frac{\rho(x)}{|x|^{n}} d x .
$$

First assume that

$$
C L \int_{A(r, 1 / 2)} \frac{\rho(x)}{|x|^{n}} d x<\frac{1}{2}
$$

By (3.11) we have

$$
p_{k} \leq 2 C L \sum_{\ell=1}^{p_{k}} \sum_{j=1}^{k} 2^{j(n-1)} \int_{Q_{i_{j}}^{j}(\ell)} \rho \circ h_{k}(x) d x .
$$

Define

$$
\Delta_{j, k}(x)=\sum_{Q \in \mathcal{Q}^{j}} S(k, Q) \chi_{Q}(x),
$$

where $S(k, Q)$ denotes the number of cubes in $\mathcal{P}_{k}$ which are descendants of $Q$. Then we may write (3.12) as (recall the notation $A_{j}$ given in (3.8))

$$
p_{k} \leq 2 C L \sum_{j=1}^{k} 2^{j(n-1)} \int_{A_{j}}\left(\rho \circ h_{k}\right)(x) \Delta_{j, k}(x) d x .
$$


Applying Hölder's inequality and the pointwise inequality (3.4) gives

$$
\begin{aligned}
p_{k} \leq & 2 C L \sum_{j=1}^{k} 2^{j(n-1)} \int_{A_{j}}\left(\rho \circ h_{k}\right)(x) \Delta_{j, k}(x) d x \\
\leq & 2 C L \sum_{j=1}^{k} 2^{j(n-1)}\left\|\hat{\rho} \circ h_{k}\right\|_{n, A_{j}}\left(\int_{A_{j}} K_{f}\left(h_{k}(x)\right)^{1 /(n-1)} \Delta_{j, k}^{n /(n-1)}(x) d x\right)^{(n-1) / n} \\
\leq & 2 C L \sum_{j=1}^{k} 2^{j(n-1)}\left\|\hat{\rho} \circ h_{k}\right\|_{n, A_{j}}\left(\max _{Q \in \mathcal{Q}^{j}} S(k, Q)\right)^{1 / n} \\
& \cdot\left(\int_{A_{j}} K_{f}\left(h_{k}(x)\right)^{1 /(n-1)} \Delta_{j, k}(x) d x\right)^{(n-1) / n} \cdot
\end{aligned}
$$

Moreover, by the definition of $\Delta_{j, k}$, we have

$$
\int_{A_{j}} K_{f}\left(h_{k}(x)\right)^{1 /(n-1)} \Delta_{j, k}(x) d x \leq p_{k} 2^{-j n} \max _{Q \in \mathcal{Q}^{j}} f_{Q} K_{f}\left(h_{k}(x)\right)^{1 /(n-1)} d x
$$

where $f$ denotes integral average. Invoking Jensen's inequality with the convex function $t \mapsto \exp \left(\lambda t^{n-1}\right)$, we see that for any $Q \in \mathcal{Q}^{j}$,

$$
f_{Q} K_{f}(h(x))^{1 /(n-1)} d x \leq \lambda^{-1 /(n-1)} \log ^{1 /(n-1)}\left(\mathcal{K} 2^{j n}\right) .
$$

Combining the estimates, we have

$$
p_{k} \leq C L p_{k}^{(n-1) / n} \sum_{j=1}^{k}\left\|\hat{\rho} \circ h_{k}\right\|_{n, A_{j}}\left(\max _{Q \in \mathcal{Q}^{j}} S(k, Q)\right)^{1 / n} \log ^{1 / n}\left(\mathcal{K} 2^{j n}\right),
$$

where $C$ depends on $n$ and $\lambda$. We next split the sum in (3.14) into two sums which are estimated separately. Let

$$
a_{k}=k-\log _{2}\left(p_{k}^{1 /(n-1)}\right) .
$$

If $j \leq a_{k}$, we use the trivial estimate

$$
\max _{Q \in \mathcal{Q}^{j}} S(k, Q) \leq p_{k}
$$

When $j>a_{k}$, we have

$$
\max _{Q \in \mathcal{Q}^{j}} S(k, Q) \leq 2^{(k-j)(n-1)},
$$

since each cube has $2^{n-1}$ descendants at the next level. 
Combining the estimates and applying Hölder's inequality to the sums, we have

$$
\begin{aligned}
(C L)^{-1} p_{k} \leq & p_{k} \sum_{j=1}^{a_{k}}\left\|\hat{\rho} \circ h_{k}\right\|_{n, A_{j}} \log ^{1 / n}\left(\mathcal{K} 2^{j n}\right) \\
& +p_{k}^{(n-1) / n} \sum_{j=a_{k}+1}^{k}\left\|\hat{\rho} \circ h_{k}\right\|_{n, A_{j}} 2^{(k-j)(n-1) / n} \log ^{1 / n}\left(\mathcal{K} 2^{j n}\right) \\
\leq & p_{k} a_{k}^{(n-1) / n}\left\|\hat{\rho} \circ h_{k}\right\|_{n, G} \log ^{1 / n}\left(\mathcal{K} 2^{a_{k} n}\right) \\
& +p_{k}^{(n-1) / n}\left\|\hat{\rho} \circ h_{k}\right\|_{n, G} 2^{k(n-1) / n}\left(\sum_{j=a_{k}+1}^{k} 2^{-j} \log ^{1 /(n-1)}\left(\mathcal{K} 2^{j n}\right)\right)^{(n-1) / n} .
\end{aligned}
$$

There exists a universal constant $C^{\prime}>0$ such that

$$
\sum_{j=a_{k}+1}^{\infty} 2^{-j} \log ^{1 /(n-1)}\left(\mathcal{K} 2^{j n}\right) \leq C^{\prime} 2^{-a_{k}} \log ^{1 /(n-1)}\left(\mathcal{K} 2^{a_{k} n}\right)
$$

Moreover, by the definition of $a_{k}$,

$$
2^{-a_{k}}=2^{-k} p_{k}^{1 /(n-1)} .
$$

Combining the estimates gives (we may assume $\mathcal{K} \geq 1$ )

$$
1 \leq C^{\prime \prime} a_{k}^{(n-1) / n}\|\hat{\rho}\|_{n, \mathbb{R}^{n}} \log ^{1 / n}\left(\mathcal{K} 2^{a_{k}}\right) \leq C^{\prime \prime}\|\hat{\rho}\|_{n, \mathbb{R}^{n}} \log \left(\mathcal{K} 2^{a_{k}}\right)
$$

where $C^{\prime \prime}$ depends on $n$ and $\lambda$. Applying (3.15) to $a_{k}$ gives (3.9).

We are left with the case

$$
\frac{1}{2} \leq C L \int_{A(r, 1 / 2)} \frac{\rho(x)}{|x|^{n}} d x
$$

where $C$ and $L$ are as in (3.11). We can apply the pointwise inequality (3.4) and Jensen's inequality, in a similar way as above, to show that

$$
\int_{A(r, 1 / 2)} \frac{\rho(x)}{|x|^{n}} d x \leq C\|\hat{\rho}\|_{n} \log \frac{\mathcal{K}}{r},
$$

where $C$ depends on $n$ and $\lambda$. Notice that, by increasing the constant $C_{1}$ according to $r$ if needed, we may assume that the inequality (1.3) holds for all values of $s>M$ such that $r \leq \mathcal{H}^{n-1}\left(F_{s}\right)$. On the other hand, if $r>\mathcal{H}^{n-1}\left(F_{s}\right)$, then $(3.16)$ combined with (3.17) gives (3.9).

Notice that the constant $C_{2}$ there only depends on $n$ and $\lambda$, while $C_{1}$ depends on $\mathcal{K}$ as the estimate above shows, but also on $n, r$ and $\lambda$ as discussed earlier. The proof of Theorem 1.2 is complete. 


\section{Example}

We next show the sharpness of Theorem 1.1, apart from the constant $\alpha$. We first consider dimension $n=2$. Let $\eta>\lambda>0$. We define a radial stretching $f: \mathbb{R}^{2} \rightarrow \mathbb{R}^{2}$ by $f(0)=0$, and

$$
f(x)= \begin{cases}\frac{z}{|z|} \exp \left(\frac{-\log ^{2}(|z|)}{\eta}\right), & \text { when }|z| \leq 1 \\ z, & \text { when }|z|>1\end{cases}
$$

Also, let $M: \mathbb{D} \rightarrow \mathbb{H}_{R}$ be the Möbius transformation

$$
M(z)=\frac{1-z}{1+z}
$$

onto the right half plane $\mathbb{H}_{R}$. Moreover, let $B_{a}: \mathbb{C} \rightarrow \mathbb{C}, 0<a<1$, be the Beurling functions defined in the introduction. Now define a mapping

$$
G_{a}: \mathbb{D} \rightarrow \mathbb{C}, \quad G_{a}=B_{a} \circ M^{-1} \circ f \circ M .
$$

Then $G_{a}(0)=0$. Moreover, since $M^{-1} \circ f \circ M$ is one-to-one and maps $\mathbb{D}$ onto $\mathbb{D}$,

$$
\int_{\mathbb{D}} J_{G_{a}}(z) d A(z)=\int_{\mathbb{D}} B_{a}(z) d A(z) \leq \pi .
$$

The distortion of $f$ is not difficult to calculate. Outside $\mathbb{D}$ the distortion is 1 , and in $\mathbb{D} \cap \mathbb{H}_{R}$ we have

$$
K_{f}(z)=\frac{|D f(z)|^{2}}{J_{f}(z)}=\max \left\{\frac{2}{\eta} \log \left(\frac{1}{|z|}\right),\left(\frac{2}{\eta} \log \left(\frac{1}{|z|}\right)\right)^{-1}\right\},
$$

cf. [7]. Since $M$ and $B_{a}$ are conformal, we have

$$
\int_{\mathbb{D}} \exp \left(\lambda K_{G_{a}}(z)\right) d A(z)=\int_{\mathbb{H}_{R}} J_{M^{-1}}(w) \exp \left(\lambda K_{f}(w)\right) d A(w) .
$$

Applying (4.1), and our assumption $\eta>\lambda$, we see that the integral on the right is finite and only depends on $\eta / \lambda$. In particular, the integral does not depend on $a$. We conclude that there exists $\mathcal{K}=\mathcal{K}(\eta, \lambda)$ such that $G_{a} \in \mathcal{F}_{\lambda, \mathcal{K}}$ for every $0<a<1$. We now have Beurling's estimate for the functions $B_{a}$ :

$$
\mathcal{H}^{1}\left(\left\{\theta:\left|B_{a}\left(e^{i \theta}\right)\right| \geq M_{a}\right\}\right) \geq C \exp \left(-M_{a}^{2}\right),
$$

where $C>0$ is independent of $a$, and $M_{a}=\log ^{1 / 2}(1 /(1-a))$. Applying this estimate and the definition of $f$, we have a similar estimate for $G_{a}$. Namely,

$$
\mathcal{H}^{1}\left(\left\{\theta:\left|G_{a}\left(e^{i \theta}\right)\right| \geq M_{a}\right\}\right) \geq C_{1} \exp \left(-\sqrt{\eta} M_{a}\right),
$$

where $C_{1}>0$ depends on the constant $C$ above and $\lambda$. Let $\gamma>0$. 
Applying (4.2), we have the following chain of inequalities:

$$
\begin{aligned}
\int_{0}^{M_{a}} \mathcal{H}^{1}\left(\left\{\left|G_{a}\left(e^{i \theta}\right)\right| \geq t\right\}\right) e^{\gamma t} d t & \geq \int_{0}^{M_{a}} \mathcal{H}^{1}\left(\left\{\left|G_{a}\left(e^{i \theta}\right)\right| \geq M_{a}\right\}\right) e^{\gamma t} d t \\
& \geq \frac{C_{1}}{e^{\sqrt{\eta} M_{a}}} \int_{0}^{M_{a}} e^{\gamma t} d t=\gamma^{-1} C_{1} e^{(\gamma-\sqrt{\eta}) M_{a}}
\end{aligned}
$$

This and Cavalieri's principle together imply

$$
\sup _{0<a<1} \int_{0}^{2 \pi} \exp \left(\hat{\alpha}\left|G_{a}\left(e^{i \theta}\right)\right|\right) d \theta=\infty
$$

whenever $\hat{\alpha}>\sqrt{\lambda}$.

In dimensions $n \geq 3$, examples showing the sharpness of Theorem 1.1, except for the constant $\alpha$, can be constructed in the same way. Namely, replacing $f$ with

$$
f(x)= \begin{cases}\frac{x}{|x|} \exp \left(\frac{-|\log | x||^{n /(n-1)}}{\eta}\right), & \text { when }|x| \leq 1 \\ x, & \text { when }|x|>1\end{cases}
$$

with a large $\eta$, and the Beurling functions $B_{a}$ with the quasiconformal logarithm maps sending $a e_{n}$ to infinity. The Möbius transformations are chosen so that they map the unit ball onto a half-space. We leave the details to the interested reader.

\section{Egg-yolk principle and Moser's inequality}

In [10] Marshall conjectured and egg-yolk principle whose validity would simplify his proof of Theorem A. The conjecture was proved in [13] by Poggi-Corradini. In [14], the following generalization was established and applied to prove Theorem B.

Theorem D ([14], Theorem 1.6). Let $n \geq 2$. There exists $0<r_{0}(n, K)<1$ such that, if $f: \mathbb{B}^{n} \rightarrow \mathbb{R}^{n}$ is a $K$-quasiregular mapping and $f(0)=0$, then $0 \leq M<$ $\max _{|x| \leq r_{0}}|f(x)|$ implies

$$
\int_{\{|f(x)|<M\}} J_{f}(x) d x \geq \alpha_{n} M^{n} .
$$

We notice that the following generalization holds.

Theorem 5.1. Let $n \geq 2$. There exists $0<r_{0}(n, \lambda, \mathcal{K})<1$ such that if $f: \mathbb{B}^{n} \rightarrow \mathbb{R}^{n}$ satisfies (1.2) and $f(0)=0$, then $0 \leq M<\max _{|x| \leq r_{0}}|f(x)|$ implies

$$
\int_{\{|f(x)|<M\}} J_{f}(x) d x \geq \alpha_{n} M^{n} .
$$


One of the main tools in the proof of Theorem D is Poletsky's inequality, which says that if $f$ is $K$-quasiregular and $\Gamma$ is a path family, then

$$
\operatorname{Mod}(f(\Gamma)) \leq K^{n-1} \operatorname{Mod}(\Gamma) .
$$

If $f$ has exponentially integrable distortion, we have the corresponding inequality in the form

$$
\operatorname{Mod}(f(\Gamma)) \leq \operatorname{Mod}_{K^{n-1}}(\Gamma) .
$$

This inequality as well as the following estimate are proved in [8].

Lemma 5.2. Let $f$ satisfy (1.2). Let $0<4 r<R<1$. Then there exist constants $C_{1}, C_{2}>0$ depending on $n, \lambda$ and $\mathcal{K}$ such that

$$
\operatorname{Mod}_{K^{n-1}}(\Gamma) \leq C_{1}\left(\int_{2 r}^{R / 2} \frac{d s}{s \log \left(C_{2} s^{-n}\right)}\right)^{1-n}
$$

where $\Gamma$ is the family of all paths connecting $\overline{B^{n}(0, r)}$ to $\mathbb{R}^{n} \backslash B^{n}(0, R)$.

Theorem 5.1 can be proved by following the proof of Theorem 1.6 in [14] and replacing the estimates there by the estimates above. We omit the details.

As discussed earlier, we do not know the optimal constant $C_{2}$ in Theorem 1.2. However, a Moser-type result can still be established as follows.

Theorem 5.3. Assume that Theorem 1.2 holds with constant $C_{2}=\beta$. Then Theorem 1.1 holds with constant $\alpha=\beta \alpha^{-1 / n}$, i.e.

$$
\sup _{f \in \mathcal{F}_{\lambda, \mathcal{K}}} \int_{\mathbb{S}^{n-1}} \exp \left(\beta \alpha_{n}^{-1 / n}|\bar{f}(\xi)|\right) d \xi<\infty .
$$

Recall that the proof of Theorem 1.1 in Section 2 gives the theorem with $\alpha<$ $\beta \alpha_{n}^{-1 / n}$. Theorem 5.3 shows that integrability still holds at the critical exponent. We need the following generalization of Moser's inequality. This is a corollary of Theorem 3 in [9].

Theorem E. Let $\psi:[0, \infty[\rightarrow[0, \infty[$ be a strictly increasing local Lipschitz function satisfying $\psi(0)=0$ and

$$
\int_{0}^{\infty}\left(\psi^{\prime}(t)\right)^{n} d t \leq 1
$$

Then there is a constant $C_{n}$ depending only on $n$ such that

$$
\int_{0}^{\infty} \exp \left(\psi(t)-t^{(n-1) / n}\right) d t^{(n-1) / n} \leq C_{n}
$$

Proof of Theorem 5.3. Cavalieri's principle yields

$$
\int_{\mathbb{S}^{n-1}} \exp \left(\beta \alpha_{n}^{-1 / n}|\bar{f}(\xi)|\right) d \xi=n \alpha_{n}+\beta \alpha_{n}^{-1 / n} \int_{0}^{\infty} \mathcal{H}^{n-1}\left(F_{s}\right) e^{\beta \alpha_{n}^{-1 / n} s} d s
$$


Choose $r_{0}$ as in Theorem 5.1, and let $M=\max _{|x| \leq r_{0}}|f(x)|$. Then, by (1.1) and Theorem 5.1, we have $M<1$ and

$$
\int_{\left\{x \in \mathbb{B}^{n}:|f(x)| \leq M\right\}} J_{f}(x) d x=\int_{0}^{M} \mathcal{A}_{n-1} f\left(E_{t}\right) d t \geq \alpha_{n} M^{n} .
$$

Again, we may assume that $f$ is continuous up to the boundary. By Theorem 1.2 and by (5.1) it suffices to estimate

$$
\int_{0}^{\|f\|_{\infty}} \exp \left(\beta \alpha^{-1 / n} s-\varphi^{(n-1) / n}(s)\right) d s,
$$

where $\varphi(s)=0$ when $0<s \leq M$ and

$$
\varphi(s)=\beta^{n /(n-1)} \int_{M}^{s} \frac{d t}{\left(\mathcal{A}_{n-1} f\left(E_{t}\right)\right)^{1 /(n-1)}}
$$

for $s>M$. Define $\tilde{\varphi}:(0, \infty) \rightarrow(0, \infty)$,

$$
\tilde{\varphi}(s)= \begin{cases}\mu s, & \text { if } 0<s \leq M \\ \varphi(s)+\mu M, & \text { if } s>M\end{cases}
$$

Here

$$
\mu=\left(\frac{\beta^{n} M}{\int_{0}^{M} \mathcal{A}_{n-1} f\left(E_{t}\right) d t}\right)^{1 /(n-1)} .
$$

Notice that $\tilde{\varphi}(0)=0$ and $\tilde{\varphi}^{\prime}(s)>0$ for every $s>0$. Furthermore,

$$
\tilde{\varphi}^{(n-1) / n} \leq \varphi^{(n-1) / n}+(\mu M)^{(n-1) / n} .
$$

Using this and (5.2) it suffices to estimate the integral

$$
\int_{0}^{\|f\|_{\infty}} \exp \left(\beta \alpha^{-1 / n} s-\tilde{\varphi}^{(n-1) / n}(s)\right) d s .
$$

Let $\phi:(0, \infty) \rightarrow(0, \infty)$,

$$
\phi(y)= \begin{cases}s, & \text { if } y=\tilde{\varphi}(s) \\ \|f\|_{\infty}, & \text { if } y>\|\tilde{\varphi}\|_{\infty}\end{cases}
$$

Changing variables with $s=\phi(y)$ in (5.3) gives

$$
\int_{0}^{\infty} \exp \left(\beta \alpha^{-1 / n} \phi(y)-y^{(n-1) / n}\right) \phi^{\prime}(y) d y .
$$

Integrating by parts, we see that it suffices to estimate

$$
\int_{0}^{\infty} \exp \left(\beta \alpha^{-1 / n} \phi(y)-y^{(n-1) / n}\right) y^{-1 / n} d y .
$$


Note that

$$
\beta \alpha_{n}^{-1 / n} \phi^{\prime}(y)= \begin{cases}\mu^{-1} \beta \alpha_{n}^{-1 / n}, & \text { if } 0<y \leq \mu M \\ \beta^{-1 / n} \alpha_{n}^{-1 / n}\left(\mathcal{A}_{n-1} f\left(E_{\phi(y)}\right)\right)^{1 /(n-1)}, & \text { if } y>\mu M .\end{cases}
$$

So by change of variables $y=\tilde{\varphi}(t)$, the definition of $\mu$ and (1.1) we have

$$
\begin{aligned}
\int_{0}^{\infty} & \left(\beta \alpha_{n}^{-1 / n} \phi^{\prime}(y)\right)^{n} d y \\
& =\mu^{1-n} \beta^{n} \alpha_{n}^{-1} M+\alpha_{n}^{-1} \beta^{-n} /(n-1) \int_{\mu M}^{\infty}\left(\mathcal{A}_{n-1} f\left(E_{\phi(y)}\right)\right)^{n /(n-1)} d y \\
& =\mu^{1-n} \beta^{n} \alpha_{n}^{-1} M+\alpha_{n}^{-1} \int_{M}^{\infty} \mathcal{A}_{n-1} f\left(E_{t}\right) d t \\
& =\alpha_{n}^{-1} \int_{0}^{\infty} \mathcal{A}_{n-1} f\left(E_{t}\right) d t \leq 1 .
\end{aligned}
$$

Now invoking Theorem E gives the claim. The proof is complete.

\section{References}

[1] Äkkinen, T.: Radial limits of mappings of bounded and finite distortion. J. Geom. Anal. 24 (2014), no. 3, 1298-1322.

[2] Beurling, A.: Études sur un problème de majorization. Thesis, Uppsala University, Uppsala, 1933.

[3] Chang, S.-Y.A. And Marshall, D. E.: On a sharp inequality concerning the Dirichlet integral. Amer. J. Math. 107 (1985), no. 5, 1015-1033.

[4] Dubinin, V. N.: Capacities and geometric transformations of subsets in $n$-space. Geom. Funct. Anal. 3 (1993), no. 4, 342-369.

[5] EssÉn, M.: Sharp estimates of uniform harmonic majorants in the plane. Ark. Mat. 25 (1987), no. 1, 15-28.

[6] Hencl, S. and Koskela, P.: Lectures on mappings of finite distortion. Lecture Notes in Mathematics 2096, Springer, Cham, 2014.

[7] Iwaniec, T. and Martin, G.: Geometric function theory and non-linear analysis. Oxford Mathematical Monographs, The Clarendon Press, Oxford University Press, New York, 2001.

[8] Koskela, P. And Onninen, J.: Mappings of finite distortion: capacity and modulus inequalities. J. Reine Angew. Math. 599 (2006), 1-26.

[9] Leckband, M. A.: An integral inequality with applications. Trans. Amer. Math. Soc. 283 (1984), no. 1, 157-168.

[10] Marshall, D. E.: A new proof of a sharp inequality concerning the Dirichlet integral. Ark. Mat. 27 (1989), no. 1, 131-137.

[11] Moser, J.: A sharp form of an inequality by N. Trudinger. Indiana Univ. Math. J. 20 (1970/71), 1077-1092. 
[12] Pankka, P., Poggi-Corradini, P. And Rajala, K.: Sharp exponential integrability for traces of monotone Sobolev functions. Nagoya Math. J. 192 (2008), 137-149.

[13] Poggi-Corradini, P.: Mapping properties of analytic functions on the disk. Proc. Amer. Math. Soc. 135 (2007), no. 9, 2893-2898.

[14] Poggi-Corradini, P. and Rajala, K.: An egg-yolk principle and exponential integrability for quasiregular mappings. J. Lond. Math. Soc. (2) 76 (2007), no. 2, $531-544$.

[15] Reshetnyak, Y.G.: Space mappings with bounded distortion. Translations of Mathematical Monographs 73, American Mathematical Society, Providence, RI, 1989.

[16] Rickman, S.: Quasiregular mappings. Results in Mathematics and Related Areas (3) 26, Springer-Verlag, Berlin, 1993.

[17] Vuorinen, M.: Conformal geometry and quasiregular mappings. Lecture Notes in Mathematics 1319, Springer-Verlag, Berlin, 1988.

Received December 18, 2013; revised March 13, 2014.

Tuomo Äkrinen: Department of Mathematics and Statistics, P.O. Box 35 (MaD), 40014 University of Jyväskylä, Finland.

E-mail: tuomo.s.akkinen@jyu.fi

Kai Rajala: Department of Mathematics and Statistics, P.O. Box 35 (MaD), 40014 University of Jyväskylä, Finland.

E-mail: kai.i.rajala@jyu.fi

Both authors were supported by the Academy of Finland, project \#257482. Äkkinen was supported by the Vilho, Yrjö and Kalle Väisälä foundation. 